\title{
The Design and Implemention of Subjective Questions Automatic Scoring Algorithm in Intelligent Turtoring System
}

\author{
Xia Yaowen and Li Zhiping \\ Institute of Information \\ Yunnan Normal University \\ Kunming,Yunnan Province,China \\ xywen_km@sina.com
}

\author{
Lv Saidong and Tang Guohua \\ Institute of Security \\ Yunnan Normal University \\ Kunming,Yunnan Province,China \\ lsdong_km@sina.com
}

\begin{abstract}
Automatic subjective question of marking is a key technology in the network test system. In order to solve this problem, this paper analyzes the grading teachers thinking reviewers subjective questions. Then introduce the concept of a one-way approach degree based on the nearness theory of fuzzy mathematics. Finally design a subjective question automatic scoring algorithm and give a specific algorithm achievement. It provide certain reference value of automatic scoring of subjective questions.
\end{abstract}

Keywords-subjective ratings; Automatic marking;one-way approach degree;intelligent tourting system;algorithm

\section{INTRODUCTION}

With the development and popularization of computer and network technology, many courses examination have been able to on the computer. Network Exam has not only convenient organization but also can work anytime,anywhere and can quickly and objectively given test scores .It reduce the test cost. Thus it will be the future trend of the development in large-scale examination. The ideal exam system not only include objective questions(multiple-choice question, determine the question, fill in the blank),but also include subjective questions(short answer,prove,computing,programming questions).The automatic scoring algorithm of objective questions use the student's answer and standard answer to compare. The results of this comparison are consistent, and then the answer is correct or wrong answers. It implement easy. There is not an examination system which is good to complete its automatic scoring because of the answer characteristics and complexity in the subjective question[1].The automatic scoring subjective questions related theory and knowledge which are the theory of artificial intelligence, pattern recognition, natural language understanding etc. Lot of technical question need to be solved, and thus it become a technical difficulty in the network examination system [2].

\section{ANALYSIS OF ALGORITHM}

Subjective questions in the answer are generally described by the language, but everyone has different levels of understanding of the knowledge and the expression is also inconsistent. Even if the student's answer is accurate, it is difficult to fully consistent with the standard answer. Accurate ratings of subjective questions are almost impossible just as objective questions. Analyze scoring teachers in the review the subjective questions, design algorithms and simulate scoring teachers this thinking activity. Then to analyze the students' answer and the standard answer, it can given the student's actual score.

\section{DESIGN OF ALGORITHM}

\section{A. Related Concepts}

To resolve the close degree of students' answers and the standard answer that the problem can be seen as the student answer and standard answer string. The following will to define the concept of one-way nearness.

Decomposed into an string to a single character, and they form an ordered collection is called a fuzzy set, $\mathrm{U}=\left\{\mathrm{u}_{1}, \mathrm{u}_{2}, \mathrm{u}_{3}, \cdots, \mathrm{u}_{\mathrm{n}}\right\}$ is called the domain. On the domain $\mathrm{U}$ of all fuzzy subset consisting denoted as F(U)(Also called fuzzy power set)[3].

To measure the closeness of two fuzzy sets, introduce the concept of a one-way approach degree [5].

Definition 1. Let $U=\{U 1, U 2, U 3, \cdots, U n\}, A, B \in F(U)$ 。 If the mapping $\delta: F(U) \times F(U) \rightarrow[0,1]$,satisfies the condition:

- $\quad \delta(\mathrm{A}, \mathrm{A})=1$

- $\quad \delta(\mathrm{B}, \mathrm{B})=1$

- If the A B C or A B C, then $\delta(A, B) \geqslant \delta(A, C)$ and said $\delta(A, B), A$ is close to the $B$ unidirectional closeness degree。

Definition 2. Let $A, B$ is the string, $A$ contain $n$ characters, $\delta(\mathrm{A}, \mathrm{B})$ represent $\mathrm{A}$ close to the $\mathrm{B}$ unidirectional closeness. In accordance with the order from left to right, the effective sum number of set $A$ in each element in the Set $B$ refers to $m, \delta(A, B)=m / n$. It is easy to verify that it meets the definition of a one-way approach degree.

The introduction of a one-way approach degree in the scoring of subjective questions, the standard answer string denoted by $A_{0}$, students answer string is denoted by $A, A_{0}$ and $A$ are two fuzzy sets. So the standard answer the $A_{0}$ and students answer $A$, one-way approach degree $\delta\left(\mathrm{A}_{0}, \mathrm{~A}\right)$ 。

\section{B. Anysis of Alogrith Thinking}

Generally, teachers in artificial reviewers subjective questions, first check the student answers a few score points, score points as many high scores. Then look at the student's 
answer and standard answer close, close high the high score. Finally, consider the students answer whether the language fluent, clarity and other factors appropriate to adjust the score.

Can be drawn from the above analysis, one of the main factors affect the scoring score points, and the other is close degree students answer and standard answer. Candidates answer subjective questions may require candidates to answer knowledge, the main argument of the candidates answer as the scoring basis. The standard answer to the need to take into account the diversity of the candidates answer, the standard answer is divided into a number of elementskeywords. The rating rules give the corresponding score which is based on correct answers key words requirements corresponding to the keywords and score as much as possible. The main task of marking system is to approach degree of keywords in the candidate's answer [5].In accordance with the proportion of the standard key words in the entire exam score, assess the small point, the last cumulative score of the keywords in the answer, and the appropriate text organization when considering candidates to answer the questions of the final grade.[6]

\section{Implementation of Algorithm}

According to the above algorithm, design function near based on VBScript [7]. The function is calculated a way close degree close to the $b$. The code of function as follows:

$$
\begin{aligned}
& \text { Function near(a,b) } \\
& \mathrm{m}=0 \\
& \mathrm{ml}=0 \\
& \mathrm{a}=\text { Trim(a) } \\
& \text { do while(len(a)>0) } \\
& \text { a asc=asc(a) } \\
& \text { if(a asc }>255) \\
& \mathrm{k}=2 \\
& \text { else } \\
& \mathrm{k}=1 \\
& \text { end if } \\
& \mathrm{a} \text { char=left(a,k) } \\
& \mathrm{a}=\mathrm{mid}(\mathrm{a}, \mathrm{k}, \mathrm{len}(\mathrm{a})-\mathrm{k}) \\
& \mathrm{l}=\mathrm{strcomp}(\mathrm{b}, \mathrm{a} \text { char) } \\
& \text { if }(\mathrm{l}>0) \\
& \quad \mathrm{m}=\mathrm{m}+1 \\
& \text { else } \\
& \mathrm{m} 1=\mathrm{m} 1+1 \\
& \text { end if } \\
& \text { loop } \\
& \mathrm{i}=\mathrm{m} /(\mathrm{m}+\mathrm{m} 1) \\
& \text { near=i } \\
& \text { End Function }
\end{aligned}
$$

\section{End Function}

\section{Subjective Questions Score}

Consider the two factors that are degree of knowledge points and close come to the subjective question of marking as shown in Equation 1:

$$
S=\left(P \times \delta_{a}\left(A_{0}, A\right)+(1-P) \times \frac{\sum_{i=1}^{n} E\left(K_{i}, A\right)}{n} \times S_{0}\right.
$$

Each symbol is the following meaning:

S: The student's actual score. Calculated based on the student respondents saved to the library.

$\mathrm{S}_{0}$ : Exam scores. The generated test paper when read from the papers in the library.

A: The student's actual answer. Students turned in their poems saved in student respondents' library.

$\mathrm{A}_{0}$ : Standard answer of the questions. Generated when the topic, read form the questions.

$\mathrm{P}$ : The proportion of share of key words in the subject scores, $0 \leqslant \mathrm{P} \leqslant 1$. The test paper generated also be rated modify, read form the papers in the library.

1-P: The proportion occupied score of the factors other than the keywords in the subject.

Calculated by the program:

$\mathrm{K}_{\mathrm{i}}$ : The keywords no $\mathrm{i}, 0 \leqslant \mathrm{i} \leqslant \mathrm{n}$. By the program split based on the keywords in the exam;

$\mathrm{E}\left(\mathrm{K}_{\mathrm{i}}, \mathrm{A}\right)$ : A one -way approach degree of keywords student answers the $\mathrm{i}, 0 \leqslant \mathrm{i} \leqslant \mathrm{n}$.Calculated by the program. $\mathrm{E}(\mathrm{K}, \mathrm{A})$ : A one-way approach degree of keywords with students answer the threshold.

$\delta_{\mathrm{a}}\left(\mathrm{A}_{0}, \mathrm{~A}\right)$ : A one-way approach degree of the standard answer and student answer

\section{DESIGN OF SYSTEM}

\section{A. Process of System}

It is easy to realize the comparison between the results of its operation with the standard answers determination score. However, when the result of the program and the standard answer does not match or unable to compile and run, then how to detect its program ideas are the main points of the automatic scoring system. The design process of the system is shown in Figure 1.

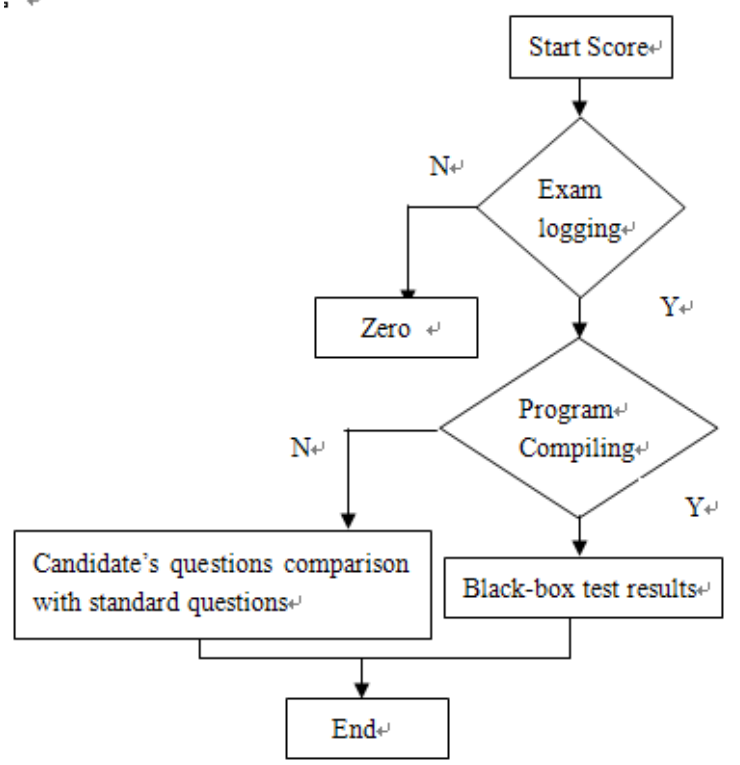

Figure 1. the process of system 


\section{B. Design of System}

Server-side environment of system is Windows Server 2003 and Internet Information Server, database of system is SQL Server2000. Based on above ideas we develop a system which is based on the Microsoft Visual Studio.NET Platform, using Visual Basic.NET as development language and database is SQL Server 2005. This system is shown in Figure 2, Figure 3 , Figure 4 and Figure 5.

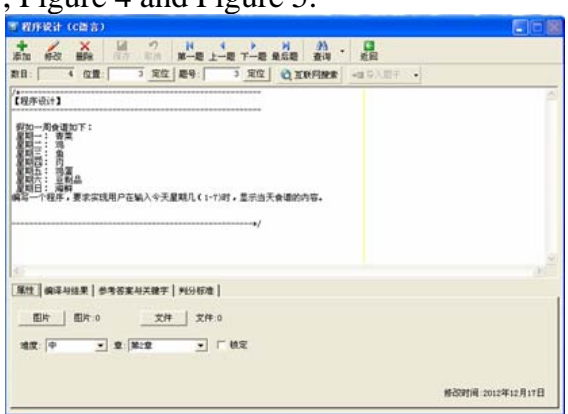

Figure 2. Main interface of system



Figure 3. Compiling interface

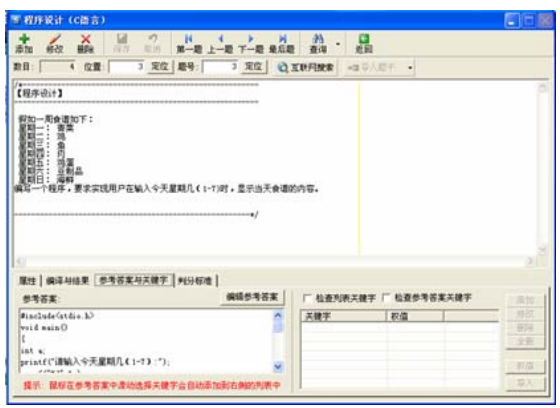

Figure 4. Key words interface

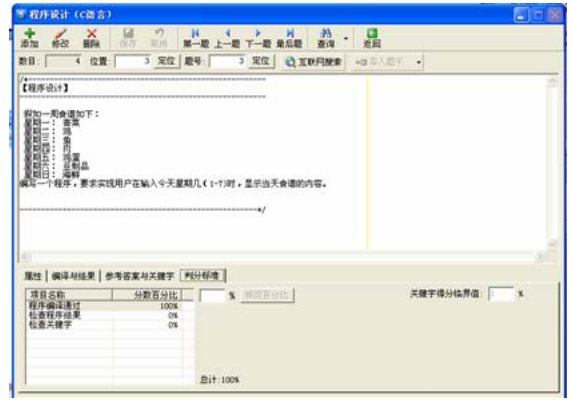

Figure 5. Assessing standards

\section{CONCLUSIONS}

The automatic scoring rating is the basic function and inevitable choice of the network test system. Automatic scoring of subjective questions is a key technology in the network test system. In the development process, it should answer the characteristics of different kinds of questions. Design and select the most appropriate scoring policy in order to achieve fast, accurate automatic scoring and reduce the adverse effects of human factors. Thus it can promote the standardized and scientific examination. In this paper, we discuss a one-way approach degree in detail and automatic scoring algorithm of subjective question. Then design algorithm. Preliminary it achieve the goal of the system, however, for accurate ratings of subjective questions also need to solve many technical problems in the future.

\section{ACKNOWLEDGMENT}

This work is supported by the National NSF of China (No.60903131), the Natural Science Fund (NSF) of Yunnan Province (No.2004F0017Q, 2005F0022Q, 2009ZC0052M).

\section{REFERENCES}

[1] Dong Yingbin,ZhuCui.Examination system based on the new $\begin{array}{llll}\text { computer network computer [J].Computer } & \end{array}$ Engineering,2001.27(8):150-152

[2] Gao Sidan,Yuan Chunfeng.Sentence similarity measurement in subjective questions automatically marking technology[J].Computer Engineering and Application,2004,14(5),132-135

[3] Zhang Sen,Weu Ming.Fuzzy query database technology[J].Electronics and automation.2000(5):23-24

[4] Meng Aiguom,Bo Shengxian.The design and implementation of a network test system subjective questions automatic scoring algorithm[J].Computer and digital engineering,2005,33(7),147-150

[5] Peng Zuzeng,Sun Yunyu.Fuzzy mathematics and its application[M].Wuhan University Press,2002.

[6] Kuang Shanyun.The design and implementation of subjective questions in network course[J].China Audio-Visual Education,2004(3).

[7] Zhao Jianmin,Guo Qing.Visual Basic 6.0 programming guide[M].Beijing: Aviation Industry Press,1999. 